\title{
Superconducting properties of very high quality NbN thin films grown by pulsed laser deposition
}

\author{
Serhii Volkov, Maros Gregor, Tomas Roch, Leonid Satrapinskyy, \\ Branislav Grančič, Tomas Fiantok, Andrej Plecenik ${ }^{*}$
}

\begin{abstract}
In this work, we study the effect of the various substrates on the growth and superconducting properties of NbN thin films grown by using pulsed laser ablation in a $\mathrm{N}_{2}+1 \% \mathrm{H}_{2}$ atmosphere on $\mathrm{MgO}, \mathrm{Al}_{2} \mathrm{O}_{3}$ and $\mathrm{Si}$ substrates. Structural and superconducting analyses of the films demonstrate that using $\mathrm{MgO}$ and $\mathrm{Al}_{2} \mathrm{O}_{3}$ substrates can significantly improve the film properties compared to $\mathrm{Si}$ substrate. The X-ray diffraction data indicate that $\mathrm{MgO}$ and $\mathrm{Al}_{2} \mathrm{O}_{3}$ substrates produce highly oriented superconducting $\mathrm{NbN}$ films with large coherent domain size in the out-of plane direction on the order of layer thickness and with a superconducting transition temperature of $13.1 \mathrm{~K}$ and $15.2 \mathrm{~K}$, respectively. On the other hand, the $\mathrm{NbN}$ film grown on the Si substrate exhibits random polycrystalline orientation. Together with the smallest coherent domain size it leads to the lower critical temperature of $8.3 \mathrm{~K}$. Finally, by using a passivation surface layer we are able to improve superconducting properties of $\mathrm{NbN}$ thin film and we observe superconducting transition temperature $16.6 \mathrm{~K}$, the one of the highest value reported so far for $50 \mathrm{~nm}$ thick $\mathrm{NbN}$ film on sapphire.
\end{abstract}

K e y w or d s: high-quality NbN films, deposition of niobium nitride, pulsed laser deposition of NbN films

\section{Introduction}

Despite of great efforts at investigation of high-temperature superconductors, the classical superconductors, such as niobium nitride $(\mathrm{NbN})$, are still attractive in many applications. $\mathrm{NbN}$ is widely used in superconducting electronics circuits, quantum computing and highfrequency devices [1] (such as high-sensitive single-photon detectors and SQUID systems) due to relatively high superconducting critical temperature $\left(T_{\mathrm{c}}\right)$ near $16 \mathrm{~K}$, energy gap $\Delta \approx 2.46 \mathrm{meV}$ in the fcc phase [2] and high upper critical magnetic field $\mathrm{B}_{c 2} \sim 40 \mathrm{~T}$.

$\mathrm{NbN}$ can be formed in hexagonal, tetragonal, or cubic crystal structures [3]. Due to this polymorphism and a high affinity to oxygen, the $\mathrm{NbN}$ is very sensitive to the deposition conditions and to achieve very good superconducting properties of thin films (below $50 \mathrm{~nm}$ ) with the high $T_{\mathrm{c}}$ is still a great challenge.

$\mathrm{NbN}$ thin films are generally fabricated by reactive magnetron sputtering [4], high-temperature chemical vapor deposition technique (HTCVD) [5] or atomic layer deposition (ALD) [6]. Olaya et. al. [4] deposited NbN films using the reactive magnetron sputtering at the substrate temperature up to $650^{\circ} \mathrm{C}$ and they showed that by increasing of the grain size from 15 to $35 \mathrm{~nm}$, the critical temperature increase from 12.6 to $16 \mathrm{~K}$ for relatively thick $1.8 \mu \mathrm{m} \mathrm{NbN}$ sample. Hazra et. al. [5] fabricated $40 \mathrm{~nm}$ thin $\mathrm{NbN}$ film with $T_{\mathrm{c}}=16.8 \mathrm{~K}$ at sapphire by using of high-temperature chemical vapor de- position technique with the high substrate temperature $\left(T_{\mathrm{s}}\right)$ of $1300^{\circ} \mathrm{C}$, which makes this technology unusable for the preparation of heterostructures. On the other hand, Linzen [6] reported the fabrication of high quality $40 \mathrm{~nm}$ thick NbN film deposited by plasma-assisted ALD with relatively lower substrate temperature of $T_{\mathrm{s}}=350{ }^{\circ} \mathrm{C}$ on sapphire, but with lower $T_{\mathrm{c}}$ of $13.8 \mathrm{~K}$.

Recently, a lot of investigations have been carried out to fabricate thin NbN films by using pulsed laser deposition (PLD) mostly on the $\mathrm{MgO}$ substrate with relatively high $T_{\mathrm{s}}$ and a middle laser fluency about $6 \mathrm{~J} \mathrm{~cm}^{-2}$ [7-9]. In this case, for $40-50 \mathrm{~nm}$ thick film, the critical temperature $T_{\mathrm{c}}$ about $16.1 \mathrm{~K}$ has been achieved. $[7,8]$. On the other hand, Kaul et. al. [9] deposited $40 \mathrm{~nm}$ thick NbN film on $\mathrm{MgO}$ and $\mathrm{Si}$ substrates with $T_{\mathrm{c}}=16.2 \mathrm{~K}$ and $T_{\mathrm{c}}=12 \mathrm{~K}$ at the room temperature but with the high laser fluency of $20 \mathrm{~J} \mathrm{~cm}^{-2}$.

In this work, we compare the structure and superconducting properties of $50 \mathrm{~nm}$ thick $\mathrm{NbN}$ films grown on various substrates by PLD at the low laser fluency mode (smaller than $5 \mathrm{~J} \mathrm{~cm}^{-2}$ ) and with the substrate temperature up to $600^{\circ} \mathrm{C}$. Using of the low laser fluency and relatively low substrate temperature allows us to prepare high quality smooth films in multilayer deposition in-situ (heterostructures), without an interdifusion between layers, which can be very useful for cryoelectronic applications.

*Department of Experimental Physics, Faculty of Mathematics, Physics and Informatics, Comenius University in Bratislava, Mlynska dolina F2, 842 48, Bratislava, Slovakia, serhii.volkov@fmph.uniba.sk 


\section{Experimental methods}

The pulsed laser deposition of NbN films were performed in an ultra-high vacuum chamber equipped with a load-lock vacuum chamber for loading and preliminary heating of samples. A pumping system consisting of turbomolecular and scroll pumps provides the background pressure of about $8 \times 10^{-7} \mathrm{~Pa}$. The deposition was carried out using an excimer $\mathrm{KrF}$ laser with the wavelength of $248 \mathrm{~nm}$ in pulse regime, pulse duration of $35 \mathrm{~ns}$, and laser fluency of $4.94 \mathrm{~J} \mathrm{~cm}^{-2}$. All $\mathrm{NbN}$ thin films were subsequently deposited from 2 inch $\mathrm{Nb}$ target (99.9\%) in the $\mathrm{N}_{2}+1 \% \mathrm{H}_{2}$ reactive atmosphere with the pressure of 9.3 $\mathrm{Pa}$ at gas flow of $80 \mathrm{sccm}$ on heated $\mathrm{MgO}$ (001), $\mathrm{Al}_{2} \mathrm{O}_{3}$ (0001) and Si (001) substrates. Substrates were ultrasonically cleaned in acetone, isopropanol and deionized water. The substrate preparation process also includes short pre-annealing in the load-lock vacuum chamber and subsequently annealing in the deposition chamber up to $600^{\circ} \mathrm{C}$. Before $\mathrm{NbN}$ deposition, the $\mathrm{Nb}$ target surface was cleaned of oxygen by using 2000 extra laser pulses. During $\mathrm{NbN}$ deposition the substrates temperature was kept constant at $600^{\circ} \mathrm{C}$.

The NbN film deposited on the $\mathrm{Al}_{2} \mathrm{O}_{3}$ substrate was in situ covered by $50 \mathrm{~nm} \mathrm{NiCu}$ layer for protection against oxidation. PLD growth of $\mathrm{NiCu}$ layer was performed in the pure $\mathrm{Ar}$ atmosphere at the pressure of $5 \mathrm{~Pa}$. The substrate temperature was kept constant at $200^{\circ} \mathrm{C}$ and the laser fluency was $6.42 \mathrm{~J} \mathrm{~cm}^{-2}$.

After deposition, $\mathrm{NbN}$ thin films were characterized by several analytical techniques to determine their crystal structure, chemical composition, morphology and electrical properties. Chemical composition of deposited samples was determined by energy dispersive spectroscopy system (EDS, Bruker, resolution $129 \mathrm{eV}$ ) at a scanning electron microscope (Tescan, Lyra) operated at energy 10 $\mathrm{keV}$. The crystal structure measurement was performed by using of PANalytical X'Pert PRO MRD X-ray diffractometer with $\mathrm{Cu} \mathrm{K} \alpha$ radiation operated at $45 \mathrm{kV}$ and 40 $\mathrm{mA}$ applying setups for symmetric $\omega / 2 \theta$-scans, rocking curves $(\omega$-scans) and grazing incidence $2 \theta$ scanning. The biaxial texture relationships between crystal lattice of deposited films and monocrystalline substrates were determined using azimuthal scans of tilted samples. Thickness of thin films was determined by X-ray reflectivity using the same diffractometer with narrow parallel beam setup. The surface morphology was analyzed by atomic force microscope (AFM, Solver 47, NT-MDT) operated in semicontact mode, with a tip curvature radius of $10 \mathrm{~nm}$. Afterwards, $5 \mu \mathrm{m}$ wide microbridges were formed from the prepared thin films using a combination of optical lithography and Ar ion beam etching. On these bridges, resistivity vs. temperature $(\rho(T))$ characteristics were then measured using standard DC four-probe measurements in a transport LHe Dewar container by utilizing Keithley 202 current source and Keithley 2000 multimeter.

\section{Results and discussions}

We performed XRD analysis of $\mathrm{NbN}$ films prepared on c-cut $\mathrm{MgO}$ (001), $\mathrm{Al}_{2} \mathrm{O}_{3}$ (0001) and on $\mathrm{Si}$ (001). Samples show fcc $\delta$-NbN cubic structure (\#01-071-0162; ICDD 1010 , nominal lattice parameter $\left.a_{0}=0.4394 \mathrm{~nm}\right)$. Thickness values of $50 \mathrm{~nm}$ for the $\mathrm{NbN}$ layer and $50 \mathrm{~nm}$ for $\mathrm{NiCu}$ passivation layer were determined by X-ray reflectivity.

XRD $\theta / 2 \theta$ pattern from sample $\mathrm{A}(\mathrm{NbN}$ on $\mathrm{MgO}$, Fig. 1(a)) shows only very narrow and relatively strong NbN 002 and its higher order NbN 004 diffractions, which implies very high (001) preferential orientation of $\mathrm{NbN}$ on $\mathrm{MgO}$. Diffractions NbN 111 and NbN 222 for sample B (NbN on $\mathrm{Al}_{2} \mathrm{O}_{3}$, Fig. 1(b)) have an order of magnitude higher intensity than 002 and 004 peaks for sample A, which means very strong (111) preferential orientation of $\mathrm{NbN}$ on $\mathrm{Al}_{2} \mathrm{O}_{3}$. Rocking curve peaks (not shown in this paper) measured around $\mathrm{NbN}$ reflections for sample $\mathrm{A}$ were order of magnitude broader $\left(\mathrm{FWHM} \sim 0.9^{\circ}, \mathrm{NbN}\right.$ 002 and 004) than for sample B $\left(\mathrm{FWHM}<0.1^{\circ}, \mathrm{NbN}\right.$ 111 and 222). After subtracting instrumental broadening from the peak widths in rocking $\omega$-scans and by converting into reciprocal space units $\Delta \mathrm{Q}_{\mathrm{x}}$ we were able to estimate lateral correlation length of mosaic domains. Lateral correlation length, meaning the dimension of the coherent mosaic domains in plane parallel to sample surface, is on the order of $30 \mathrm{~nm}$ for film A and more than $200 \mathrm{~nm}$ for sample B respectively. The $\mathrm{NbN}$ films A and B show only single fcc $\delta$-NbN oriented phase and did not contain any detectable polycrystalline random fraction as checked by grazing incidence X-ray diffraction flat patterns without polycrystalline peaks. This also invoked strong expectation of the biaxial texture (epitaxial mosaic blocks).

Instead of full texture pole figures the inset of Fig. 1(a) shows azimuthal $\phi$-scan at the diffraction position of $\mathrm{NbN}$ (on $\mathrm{MgO}$ ) 111 with sample normal tilted at $\psi=$ $54.6^{\circ}$ with respect to scattering plane. The epitaxial orientation relationships are as follows: $\mathrm{NbN}(001) \|$ $\mathrm{MgO}(001), \mathrm{NbN}[100] \| \mathrm{MgO}[100]$. Inset of Fig. 1(b) shows azimuthal scan through the NbN 111 diffraction of sample B tilted $70.5^{\circ}$ from surface normal. Six peaks and position of sapphire reflections (not shown) suggest orientation relationships: $\mathrm{NbN}(111) \| \mathrm{Al}_{2} \mathrm{O}_{3}(0001)$, NbN[110] $\| \mathrm{Al}_{2} \mathrm{O}_{3}[1-100]$ as sketched in Fig. 1(d). Hexagonal symmetry of $\mathrm{Al}_{2} \mathrm{O}_{3}$ surface leads to two possible variants of in-plane $\mathrm{NbN}$ orientations, which generate six observable peaks in the azimuthal scan. Average azimuthal peak width for sample B $\left(1.97^{\circ}\right)$ is twice as larger than for sample A $\left(0.97^{\circ}\right)$ which can origin from larger in-plane lattice mismatch between $\mathrm{NbN}(111)$ and $\mathrm{Al}_{2} \mathrm{O}_{3}$ (0001) or $\mathrm{X}$-ray beam defocusing effect due to larger tilt of sample B.

Grazing incidence XRD measurement of sample C (NbN on Si, Fig. 1(c)) shows random fcc $\delta$-NbN crystallites orientation. Additional peaks observed at $2 \theta=$ $62.5^{\circ}$ and $82.4^{\circ}$ are most likely related to the minor hexagonal $\mathrm{Nb}_{3} \mathrm{~N}_{4}$ phase. Using Williamson-Hall analysis of the peak widths related to fcc- $\mathrm{NbN}$ we have estimated coherent domain size of $20 \mathrm{~nm}$ and microstrain of 

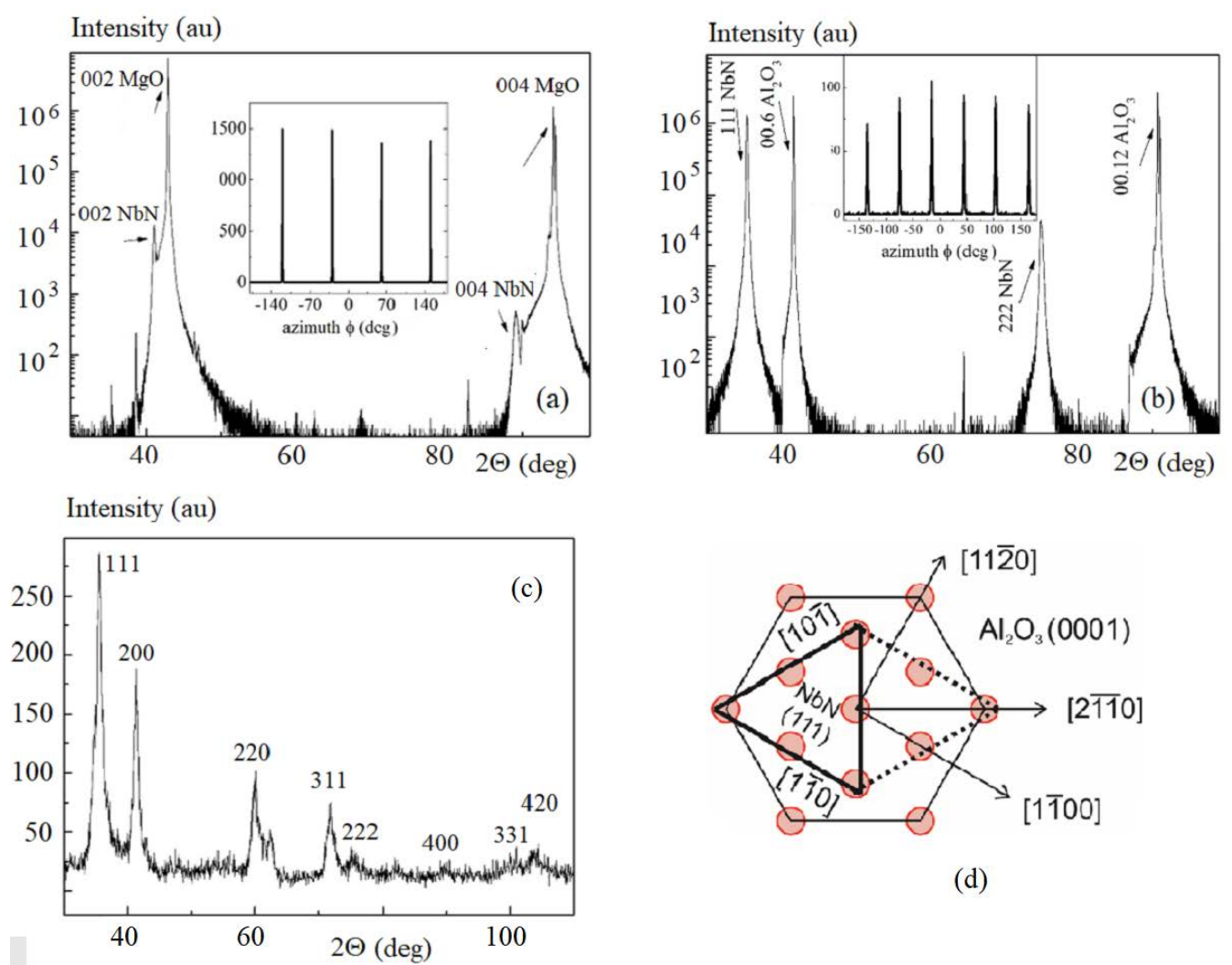

(d)

Fig. 1. XRD patterns for samples A, B and C respectively (a), (b) and (c)). Insets in the panels: (a) and (b) - the azimuthal scans at $\mathrm{NbN} 111$ diffraction, (d) - lattice relation between (0001) $\mathrm{Al}_{2} \mathrm{O}_{3}$ and (111) NbN planes with two variants (dotted lines), red circles represent surface oxygen sites.

Table 1. Analyzed parameters of NbN crystal structure

\begin{tabular}{lcccc} 
Sample & Layers configuration & $a_{0}-\mathrm{NbN}(\mathrm{nm})$ & Average crystallite lateral size $(\mathrm{nm})$ & Substrate-lattice mismatch (\%) \\
\hline $\mathrm{A}$ & $\mathrm{MgO} / \mathrm{NbN}$ & 0.4394 & 30 & 4.27 \\
$\mathrm{~B}$ & $\mathrm{Al}_{2} \mathrm{O}_{3} / \mathrm{NbN}$ & 0.4379 & $\sim 200$ & 13.1 \\
$\mathrm{C}$ & $\mathrm{Si} / \mathrm{NbN}$ & 0.4396 & 20 & -19.1 \\
$\mathrm{D}$ & $\mathrm{Al}_{2} \mathrm{O}_{3} / \mathrm{NbN} / \mathrm{NiCu}$ & 0.4383 & $\sim 170$ & 13.1 \\
\hline
\end{tabular}

$0.8 \%$. Estimating from the width of $\mathrm{NbN}$ peaks in $\theta / 2 \theta$ scans the coherent domain size in the direction normal to the film surface is on the order of the film thickness. A lot more detailed results of structural analysis will be presented in our coming next paper.

Interestingly, lateral correlation length of sample B is much larger than for sample $\mathrm{A}$ in spite of three times larger mismatch of $\mathrm{NbN}$ on $\mathrm{Al}_{2} \mathrm{O}_{3}$ (Tab. 1). Lattice mismatch $m=\left(d_{\mathrm{f}}-d_{\text {sub }}\right) / d_{\text {sub }}$ for NbN grown on $\mathrm{Al}_{2} \mathrm{O}_{3}$ uses $d$-spacings of the corresponding planes $d_{\mathrm{NbN}}=\sqrt{2} a_{\mathrm{NbN}}$ and $d_{\mathrm{Al} 2 \mathrm{O} 3}=2 / \sqrt{3} a_{\mathrm{Al} 2 \mathrm{O} 3}$. As already observed for $\mathrm{AlN}$ [10] and NbN [11], domain matching epitaxy [12] with periodic array of misfit dislocations would allow partial relaxation. In this situation every $8 \mathrm{NbN}$ atomic planes are matching 9 atomic planes of the $\mathrm{Al}_{2} \mathrm{O}_{3}$ with lattice mismatch $m=\left(8 \times d_{\mathrm{NbN}}-9 \times d_{\mathrm{Al} 2 \mathrm{O} 3}\right) /\left(9 \times d_{\mathrm{Al} 2 \mathrm{O} 3}\right)=0.5 \%$.

Resistivity $v s$ temperature dependencies of the $\mathrm{NbN}$ microbridges are presented in Fig. 2. and Tab. 2. The superconducting transition temperature $\left(T_{\text {con }}\right)$, the zero resistivity transition temperature $\left(T_{\mathrm{c} 0}\right)$ and the transition width $\left(\Delta T=T_{\text {con }}-T_{\mathrm{c} 0}\right)$ of our $\mathrm{NbN}$ thin films depend on the used substrate. It can been seen, the film deposited on the sapphire substrate exhibits the highest critical temperature $\left(T_{\text {con }}=15.5 \mathrm{~K}\right)$ with a zero resistivity transition temperature of $T_{\mathrm{c} 0}=15.2 \mathrm{~K}$ followed by the film deposited on $\mathrm{MgO}\left(T_{\mathrm{con}}=13.7 \mathrm{~K}, T_{\mathrm{c} 0}=13.1\right.$ $\mathrm{K})$. On the other hand, the $\rho(T)$ measurement of sample deposited on Si shows the worst superconducting properties with $T_{\text {con }}=10.8 \mathrm{~K}, T_{\mathrm{c} 0}=8.3 \mathrm{~K}$, and with the broadest transition width of $\Delta T=2.5 \mathrm{~K}$.

The resistivity at $20 \mathrm{~K}\left(\rho_{20}\right)$ of our epitaxial $\mathrm{NbN}$ films was in the range from 100 to $150 \mu \Omega \mathrm{cm}$ (see Tab. 2), which is higher than values $(10-50 \mu \Omega \mathrm{cm})$ reported for single-crystal NbN film [13]. Furthermore, our NbN single films showed different type of $\rho(T)$ characteristics (Fig. 2 ) with residual resistivity ratio $\mathrm{RRR}=\rho_{300} / \rho_{20}$ varying in the range from 0.82 to 1.04 . As naturally expected, the residual resistivity $\rho_{20}$ can be linked to intragrain 
Table 2. The electrical parameters of investigated NbN films

\begin{tabular}{|c|c|c|c|c|c|c|c|}
\hline Sample & Layer configuration & $\begin{array}{c}\rho_{300} / \rho_{20} \\
(-) \\
\end{array}$ & $\begin{array}{l}\text { Resistivity at } 20 \mathrm{~K}, \rho_{20} \\
(\mu \Omega \mathrm{cm})\end{array}$ & $\begin{array}{c}\text { Resistivity at } 300 \mathrm{~K}, \rho_{300} \\
(\mu \Omega \mathrm{cm})\end{array}$ & $\begin{array}{l}T_{\text {con }} \\
(\mathrm{K})\end{array}$ & $\begin{array}{l}T_{\mathrm{c} 0} \\
(\mathrm{~K})\end{array}$ & $\begin{array}{l}\Delta T_{\mathrm{c}} \\
(\mathrm{K}) \\
\end{array}$ \\
\hline$\overline{\mathrm{A}}$ & $\mathrm{MgO} / \mathrm{NbN}$ & 0.8494 & 148 & 126 & 13.7 & 13.1 & 0.6 \\
\hline B & $\mathrm{Al}_{2} \mathrm{O}_{3} / \mathrm{NbN}$ & 1.042 & 99.6 & 104 & 15.5 & 15.2 & 0.3 \\
\hline $\mathrm{C}$ & $\mathrm{Si} / \mathrm{NbN}$ & 0.8242 & 196 & 162 & 10.8 & 8.3 & 2.5 \\
\hline $\mathrm{D}$ & $\mathrm{Al}_{2} \mathrm{O}_{3} / \mathrm{NbN} / \mathrm{NiCu}$ & 1.071 & 53.8 & 57.6 & 16.6 & 16.4 & 0.2 \\
\hline
\end{tabular}
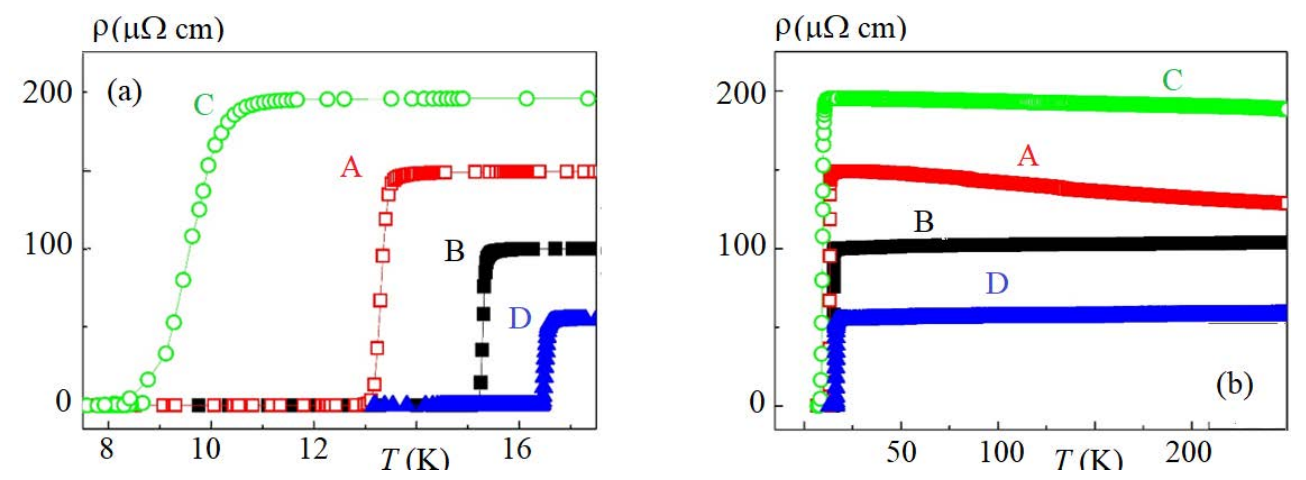

Fig. 2. $\rho(T)$ characteristics of samples: (a) - A, B, C, D viewed at low temperature range, and (b) - in wide temperature range

scattering and residual resistivity ratio reflects intergrain connectivity imperfections due to porosity and/or impurities between the grains [14]. It has been shown, that with the decrease of residual resistivity of sample, the transition temperature $T_{\mathrm{c}}$ increases [13].

As is seen in Fig. 2, only the sample B with the highest $T_{\mathrm{c}}$ shows fully metallic $\rho(T)$ characteristics with the lowest $\rho_{20}=99.6 \mu \Omega \mathrm{cm}$ and with $\mathrm{RRR}>1$. On the other hand, samples with the lower $T_{\mathrm{c}}$ (A and $\mathrm{C}$ ) show semiconductor behavior of $\rho(T)$ characteristics with higher residual resistivity, which indicates higher concentration of scattering centers inside the grains and the $R R R<1$ which implies some impurities between grains.

Even if all the samples (A, B, C) were deposited together at the same time only the sample B showed the fully metallic behavior. EDS analysis demonstrates almost the same chemical composition for all samples with $\mathrm{Nb} / \mathrm{N}$ ratio to be 1.04 , without any chemical contamination during the deposition.

These superconducting transport properties directly correlate with the crystal structure quality of $\mathrm{NbN}$. The sample $\mathrm{B}$ with the best $T_{\mathrm{c}}$ exhibits relatively high crystallite lateral size. Although this sample has the higher lattice mismatch of $13.1 \%$ than sample A (4.27\%), the domain matching effect decreases the effective lattice mismatch to only $0.5 \%$. The sample $\mathrm{C}$ with the worst $T_{\mathrm{c}}$ has the smallest crystallite lateral size with the highest lattice - substrate mismatch of $-19.1 \%$. The $T_{\mathrm{c}}$ value is related not only to lattice parameter, but also to microstructure and texture of films. For instance, the best $T_{\mathrm{c}}$ of $\mathrm{NbN}$ film prepared by magnetron sputtering was observed for $a=0.4388 \mathrm{~nm}$ with $\mathrm{Nb} / \mathrm{N}$ ratio near $1.09[3]$. On the other hand, the best $T_{\mathrm{c}}$ obtained by PLD technology on $\mathrm{MgO}$ was achieved for $a=0.4428 \mathrm{~nm}$ which is by $0.8 \%$ higher than reference value. In addition, different $T_{\mathrm{c}}$ 's were also reported for samples with almost the same stoichiometric composition [15, 16].

This effect was theoretically explained using model of the granulated superconductor as a sequence of Josephson junctions [17]. It was found, that the presence and evolution of superconductivity mainly depends on the resistivity across the grain boundaries (intergrain resistivity). As a result, we can assume that strongly-oriented thin films with the higher crystalline lateral size should have a higher $T_{\mathrm{c}}$ with fully metallic $\rho(T)$ characteristics due to lower number of grain boundaries and the stronger bonds at the low angle grain boundaries in comparison with randomly oriented films, which usually exhibit the semiconducting $\rho(T)$ behaviour. This has been corroborated by a number of works (see eg [4] and [18]) where high $T_{\mathrm{c}}$ 's were observed for highly-oriented samples with different grown directions [111] or [200].

The second important contribution to the $T_{\mathrm{c}}$ value is the coherent domain size (CDS). When the CDS is lowered to the superconducting coherence length (nanocrystaline films), it leads to suppressing of superconductivity. The $T_{\mathrm{c}}$ in these films can be dramatically reduced in the presence of just small concentration of inhomogeneitys and impurities. Thus, larger grain size means higher probability to obtain high $T_{\mathrm{c}}$ within the grains.

Another possible determinant of the superconducting transport properties of $\mathrm{NbN}$ thin film is a degradation. Degradation is mainly manifested as an oxidation progressing from the top of superconductive films starting 
(a)

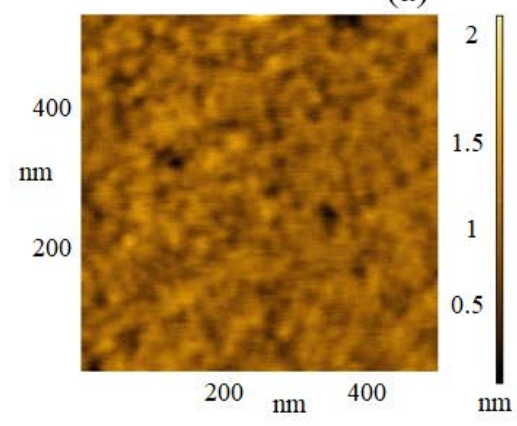

(b)

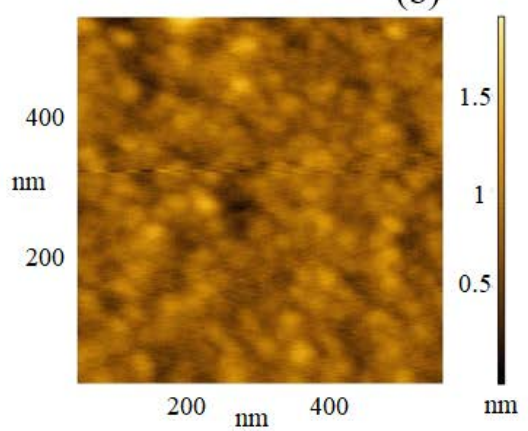

(c)

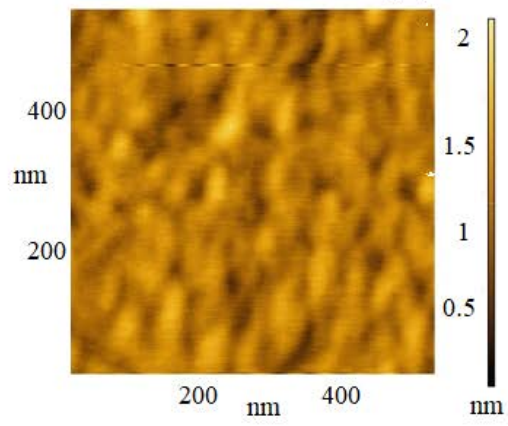

Fig. 3. Morphology of samples A, B and C ((a), (b) and (c), respectively

at the grain boundaries. It plays an extremely important role especially in the case of very thin superconductor layer because of its relatively small bulk/surface ratio. Shino et. al. obtained the increasing of $T_{\mathrm{c}}$ from 7.3 to $10.5 \mathrm{~K}$ for ultra-thin NbN films by using of AlN passivation layer [19]. In our case, we obtained the increasing of $T_{\text {con }}$ for NbN films up to $16.6 \mathrm{~K}$ by using of $\mathrm{NiCu}$ passivation layer deposited in situ on $\mathrm{NbN}$ film (Fig. 2). Despite the fact that $\mathrm{NiCu}$ film was fabricated at $T_{\mathrm{s}}=200^{\circ} \mathrm{C}$, no interdiffusion between layers was observed. It can be proved by very narrow and uniform superconductive temperature transition with the width of $0.2 \mathrm{~K}$, and it does not show any additional superconductive phases (Tab. 2, Fig. 2). We performed an additional investigation of crystal structure of $\mathrm{NbN}$ covered by $\mathrm{NiCu}$ layer in order to testify that there is essentially no change of $\mathrm{NbN}$ crystal structure during $\mathrm{NiCu}$ passivation layer deposition. There was only slight increase of lattice parameter and decrease of CDS (Tab. 1). Therefore, we can conclude that the covering of $\mathrm{NbN}$ film by $\mathrm{NiCu}$ passivation layer does not lead to the structural transformation processes, which can affect the superconducting properties of individual $\mathrm{NbN}$ layer. We believe that increasing of $T_{\mathrm{c} 0}$ is caused only by the protection of $\mathrm{NbN}$ against oxidation process.

Eventually, the atomic force microscope has been used for surface morphology analysis of deposited films (Fig. 3, Tab. 3). AFM scans on $500 \times 500 \mathrm{~nm}$ area show a root-mean-square (RMS) roughness varying from 0.17 to $0.22 \mathrm{~nm}$ and peak to peak values from 1.90 to $2.15 \mathrm{~nm}$. All samples demonstrated very small roughness with high homogeneity which permits their usage in heterostructures for various cryogenic applications.

Table 3. Morphology of $50 \mathrm{~nm} \mathrm{NbN}$ films

\begin{tabular}{lcc} 
Sample & Roughness RMS & Peak-to-Peak value \\
\hline & $(\mathrm{nm})$ & $(\mathrm{nm})$ \\
$\mathrm{A}$ & 0.17 & 1.90 \\
$\mathrm{~B}$ & 0.18 & 2.12 \\
$\mathrm{C}$ & 0.22 & 2.15 \\
\hline
\end{tabular}

\section{Conclusions}

We have shown that superconducting properties of NbN thin films prepared by PLD technique significantly depend on the used substrates. The better structural properties with the epitaxial mosaic blocks have been grown on the c-cut $\mathrm{Al}_{2} \mathrm{O}_{3}$ and $\mathrm{MgO}$ substrates compared to $\mathrm{Si}$ substrate with random grains orientation. Although $\mathrm{MgO}$ has the smallest lattice mismatch with the cubeon-cube growth trend, the larger grain size has been observed on $\mathrm{Al}_{2} \mathrm{O}_{3}$ substrate. According to the literature, the hexagonal $\mathrm{Al}_{2} \mathrm{O}_{3}$ surface may allow domain matching epitaxy with the smallest lattice mismatch of $0.5 \%$ due to partial relaxation with periodic array of misfit dislocations.

The structural considerations are directly reflected in superconducting properties. The film grown on sapphire exhibits the largest grain size with the smallest residual resistivity at $20 \mathrm{~K}$ and fully metallic $\rho(T)$ behavior which represent tight packing of grains and good contact between them. This film has the best $T_{\mathrm{c}}$ value of 15.2 K compared to films deposited on $\mathrm{MgO}$ and $\mathrm{Si}$ with the smaller grain size, the higher residual resistivity and semiconductor $\rho(T)$ behaviour, where superconducting transition temperatures of $T_{\mathrm{c} 0}=13.1 \mathrm{~K}$ and $T_{\mathrm{c} 0}=8.3$ $\mathrm{K}$, has been measured, respectively. It is in the qualitative agreement with previous theoretical works, which estimated the influence of the grain size and resistivity inside and between grains on superconducting properties.

Due to rapid degradation of $\mathrm{NbN}$ thin film, $50 \mathrm{~nm}$ thick $\mathrm{NiCu}$ passivation layer has been used, by which we were able to sufficiently improve the $T_{\mathrm{c}}$ of NbN films up to value $T_{\text {con }}=16.6 \mathrm{~K}$. Finally, our NbN thin films show very small roughness, which is crucial qualification for the preparation of heterostructures for usage in cryogenic applications.

\section{Acknowledgements}

This work was supported by the Slovak Research and Development Agency under the contract no. APVV-160315. It is also result of the project implementations: ITMS 26240220027 and 26210120010 supported by the Research \& Development Operational Program funded by 
the ERDF and grant UK/170/2018 funded by Comenius [10] Th. Kehagias, Ph. Komninou, G. Nouet, P. Ruterana, and Th. University. Karakostas, "Misfit relaxation of the $\mathrm{AlN} / \mathrm{Al}_{2} \mathrm{O}_{3}$ (0001) interface", Phys. Rev. B, vol. 64, pp. 195329, 2001.

\section{REFERENCES}

[1] D. Dochev et al, "Growth and characterization of epitaxial ultra-thin NbN films on 3C-SiC/Si substrate for teraherz applications", Supercond. Sci. Technol., vol. 24, pp. 035016, 2011.

[2] E. A. Antonova, D. R. Dzhurgaev, G. P. Motulevich and V. A. Sukhov, "Superconducting energy gap of niobium nitride", $Z$ h. Exsp. Theor. Fiz., vol. 80, pp. 2426-2429, 1981.

[3] E. I. Alessandrini, V. Sadagopan, R. B. Laibowitz, "Relationship between Structure and Sputtering Parameters in NbN Films", J. Vac. Sci. \& Technol., vol. 8, pp. 188, 1971.

[4] J. J. Olaya, L. Huerta, S. E. Rodil, R. Escamilla, "Superconducting niobium nitride films deposited by unbalanced magnetron sputtering", Thin Solid Films, vol. 516, pp. 8768-8773, 2008.

[5] D. Hazra et al, "Superconducting properties of very high quality NbN thin films grown by high temperature chemical vapor deposition", Supercond. Sci. Technol., vol. 29, pp. 105011, 2016.

[6] S. Linzen et al, "Structural and electrical properties of ultrathin niobium nitride films grown by atomic layer deposition", Sci. Technol., vol. 30, pp. 035010, 2017.

[7] A. Bhat, X. Meng, A. Wong, T. V. Duzer, "Superconducting $\mathrm{NbN}$ films grown using pulsed laser deposition for potential application in internally shunted Josephson junctions", Supercond. Sci. Technol., vol. 12, pp. 1030-1032, 1999.

[8] R. E. Treece et al, "Pulsed laser deposition of high-quality NbN thin films", Appl. Phys. Lett., vol. 65, pp. 2860, 1994.

[9] A. B. Kaul, T. D. Sands, T. V. Duzer, "High-Tc superconducting $\mathrm{NbN}$ films with low particulate density grown at $25^{\circ} \mathrm{C}$ using pulsed laser deposition", J. Mater. Res., vol. 16, pp. 1223-1226, 2001.

[11] F. Merciere et al, "Niobium nitride thin films deposited by high temperature chemical vapor deposition", Surface and Coatings Technology, vol. 260, pp. 125-132, 2014.

[12] J. Narayan, B. C. Larson, "Domain epitaxy: A unified paradigm for thin film growth", J. Appl. Phys., vol. 93, pp. 278, 2003.

[13] Z. Wang, A. Kawakami, Y. Uzawa, B. Komiyama, "Superconducting properties and crystal structures of singlecrystal niobium nitride thin films deposited at ambient substrate temperature", J. Appl. Phys., vol. 79, pp. 7837-7842, 1996.

[14] J. M. Rowell, S. Y. Xu, X. H. Zeng, A. V. Pogrebnyakov, Q. Li, X. X. Xi, J. M. Redwing, W. Tian, X. Pan, "Critical current density and resistivity of $\mathrm{MgB}_{2}$ films", Appl. Phys. Lett., vol. 83, pp. 102-104, 2003.

[15] J. R. Clem et al, "Ambegaokar-Baratoff-Ginzburg-Landau crossover effects on the critical current density of granular superconductors", Phys. Rev. B, vol. 35, pp. 6637, 1987.

[16] K. Senapati, N. K. Pandey, R. Nagar, R. C. Budhani, "Normal-state transport and vortex dynamics in thin films of two structural polymorphs of superconducting NbN", Phys. Rev. B, vol. 74, pp. 104514, 2006.

[17] B. Abeles, "Effect of charging energy on superconductivity in granular metal films", Phys. Rev. B, vol. 15, pp. 2828, 1977.

[18] S. Thakoor, J. L. Lamb, A. P. Thakoor, S. K. Khanna, "High $\mathrm{T}_{\mathrm{c}}$ superconducting $\mathrm{NbN}$ films deposited at room temperature", $J$. Appl. Phys., vol. 58, pp. 4643, 1985.

[19] T. Shino et al, "Improvement of the critical temperature of superconducting $\mathrm{NbTiN}$ and $\mathrm{NbN}$ thin films using the AlN buffer layer", Sci. Technol., vol. 23, pp. 045004, 2010.

Received 19 March 2019 